REVISTA DE LA CEPAL 83. AGOSTO 2004

\title{
Desempeño exportador de las empresas chilenas: algunos hechos estilizados
}

Roberto Alvarez E.

1

pesar de la positiva evolución del desempeño exportador chileno en las últimas décadas, las exportaciones siguen concentradas en pocos productos, pocos mercados y en un número reducido de empresas. En este trabajo se aborda este último punto, analizando el comportamiento exportador de las firmas. Los principales hechos estilizados de este fenómeno son los siguientes: sólo un grupo reducido de firmas es capaz de exportar permanentemente, y estas empresas muestran niveles de productividad, capital humano y tamaño muy superiores al resto de las empresas.

Roberto Álvarez E. 


\section{I}

\section{Introducción}

En las últimas décadas del siglo XX el sector externo de la economía chilena se caracterizó por una fuerte expansión y diversificación de sus exportaciones. La mayor diversificación no sólo se puede apreciar porque se redujo la fuerte dependencia de las exportaciones de cobre, que pasaron de representar más del $80 \%$ de las exportaciones del país a principios del decenio de 1980 a menos del $40 \%$ en la década de 1990, sino también por la creciente introducción de nuevos productos, la apertura de nuevos mercados y la entrada de un número mayor de empresas a los mercados internacionales.

Entre 1970 y 1989, el número de productos exportados se elevó de 1.200 a 1.490 y en el período 19902002 subió de 2.300 a $3.750 .{ }^{1}$ En cuanto a la apertura de nuevos mercados, se tiene que el número de países de destino de las exportaciones chilenas aumentó de 31 en 1970 a más de 150 en 2002.

Sin embargo, a pesar de la positiva evolución del desempeño exportador de Chile, que se analiza con más detalle en la sección II, los datos muestran que las exportaciones del país siguen concentradas en pocos productos, pocos mercados y en un número reducido de empresas. En efecto, el $50 \%$ de las exportaciones chilenas se concentra en 10 productos, en 5 mercados y en 25 empresas (ProChile, 2002).

Relacionado con esta alta concentración de las exportaciones en pocas empresas, mercados y productos se da un fenómeno que no ha sido estudiado en Chile y que constituye la principal motivación de este trabajo: la existencia de un grupo importante de em-

\footnotetext{
$\square$ El autor agradece los valiosos comentarios y sugerencias de Bernardita Escobar, Ronald Fischer, Pablo González, Dominique Hachette, Patricia Noda y Alejandra Sanhueza, así como también a los asistentes a los seminarios en el Departamento de Economía de la Universidad de Chile, la Universidad de California Los Angeles, y el Departamento de Investigación del Banco Interamericano de Desarrollo. Además, agradezco a Erwin Hansen, quien colaboró eficazmente en el procesamiento de la información, y a Marichu Meyer y Anthony Lemus por su valiosa ayuda en ProChile. Esta investigación contó con el financiamiento del Fondo para Políticas Públicas y ProChile.

${ }^{1}$ La cantidad de productos exportados después de 1990 no se puede comparar con la de años precedentes porque en el registro de las exportaciones se pasó en 1989 de la Nomenclatura Arancelaria de Bruselas al Sistema Armonizado de Aranceles, que es más desagregado.
}

presas que no logra consolidarse en los mercados internacionales. En efecto, casi un tercio de las firmas no logra permanecer exportando por más de un año. ${ }^{2}$

Aunque existen diversos estudios acerca del impacto de la liberalización comercial en Chile sobre el crecimiento y la productividad, ${ }^{3}$ no hay mucha evidencia respecto a las causas del dinamismo exportador. Algunas contribuciones recientes son la de Agosin (1999), quien estudia los determinantes del crecimiento de las exportaciones de manufacturas, y la de Gutiérrez de Pinheres y Ferrantino (1997), quienes analizan los factores macroeconómicos que han influido en el proceso de diversificación de las exportaciones chilenas.

No obstante, existe escasa información empírica respecto al desempeño exportador a nivel de la empresa. Como excepciones en esta materia cabe mencionar dos estudios: el de Álvarez y Crespi (2000a y 2000b), que ha investigado el impacto de los instrumentos administrados por ProChile sobre el desempeño de las empresas y ha concluido que éstos generan un efecto positivo sobre las empresas que los utilizan, incrementando el número de mercados y el valor exportado; y el de Macario (2000), que utiliza una muestra de empresas exportadoras e investiga los factores determinantes de la decisión de exportar, los potenciales beneficios que podrían internalizar las empresas exportadoras y el rol que los instrumentos de promoción han tenido sobre el dinamismo de las exportaciones.

En esta área, durante los últimos años se ha desarrollado una línea de investigación sobre los microfundamentos del desempeño exportador, impulsada por un proyecto del Banco Mundial cuyo objetivo era analizar los factores que llevan a las empresas a la decisión de exportar, así como las consecuencias de tal decisión (Banco Mundial, 1996). Una de las principales conclusiones de estos estudios es que la entrada a los mercados internacionales tiene costos irrecuperables (sunk costs), por lo cual las empresas, una vez que se transforman en exportadoras, tenderán a permanecer

\footnotetext{
${ }^{2}$ En los últimos años, esta tasa anual de salida ha tendido a mantenerse en torno al $30 \%$, salvo en 2000 , año en que fue de casi $40 \%$ (véase detalles más adelante, en el cuadro 8).

${ }^{3}$ Como los de Rojas, López y Jiménez (1997), García, Meller y Repetto (1996), Fuentes (1995), Figueroa y Letelier (1994), Marshall (1992) y Tybout, De Melo y Corbo (1991).
} 
exportando aunque las condiciones se vuelvan desfavorables (Roberts y Tybout, 1997; Bernard y Jensen, 2001). Por lo señalado en un párrafo anterior, el caso chileno contradice esta aseveración. En efecto, un grupo importante de empresas que empiezan a exportar no persiste en esta actividad.

En tal sentido, el objetivo de este trabajo es analizar cuáles son los principales hechos estilizados del comportamiento exportador de las empresas chilenas y extraer algunas conclusiones respecto a los princi- pales factores que llevan a tener éxito en los mercados internacionales.

A continuación, la sección II analiza los antecedentes relativos al sector exportador chileno. La sección III examina las características de las empresas exportadoras y no exportadoras. La sección IV presenta la fuente de los datos y resume los principales hechos estilizados del desempeño exportador de las empresas en el decenio de 1990 y la sección V ofrece las principales conclusiones.

\section{II}

\section{Antecedentes del sector exportador chileno}

\section{Crecimiento y diversificación de las exporta- ciones}

Uno de los principales beneficios de la apertura comercial iniciada en el decenio de 1970 fue la reducción del sesgo antiexportador implícito en el modelo de sustitución de importaciones. La racionalización de la compleja estructura de barreras comerciales y la aplicación de ciertas políticas de fomento permitieron un mayor crecimiento y diversificación de las exportaciones chilenas. Entre esas políticas cabe destacar la introducción del reintegro simplificado, el uso de programas de conversión de deuda que facilitaron la entrada de la inversión extranjera en el sector exportable, la participación activa del Estado en el suministro de información, los subsidios en el sector forestal y la política cambiaria a partir de 1982 (Agosin, 1999).

Como se aprecia en el gráfico 1, antes de la apertura comercial las exportaciones crecieron muy lentamente. No obstante, desde mediados del decenio de 1980 el crecimiento del sector exportador ha sido muy superior al de las décadas anteriores. En efecto, entre 1960 y 1973 el crecimiento medio anual de las exportaciones fue de 3,5\% y en el período 1973-1983 alcanzó una tasa similar, de 3,6\% al año. Sin embargo, fue entre 1983 y 2002 que se observó el más rápido crecimiento del sector exportador, con una tasa media anual de $5,5 \%{ }^{4}$

Este crecimiento de las exportaciones fue acompañado por un significativo aumento de su diversifica-

\footnotetext{
${ }^{4}$ Véase en Ffrench-Davis (2002) un análisis de la relación entre exportaciones y crecimiento en Chile.
}

GRAFICO

Chile: Exportaciones, 1960-2002 ${ }^{\mathrm{a}}$ (Indice: $1960=100$ )

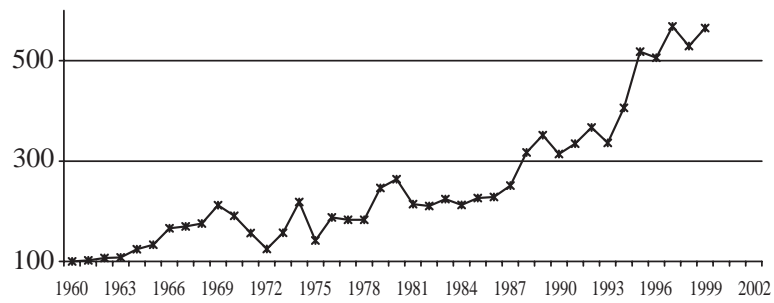

Fuente: Elaboración propia sobre la base de cifras del Banco Central de Chile.

a Las exportaciones nominales fueron deflactadas de acuerdo al
Índice de Precios Externos que elabora el Banco Central de Chile.

ción. ${ }^{5}$ Como se aprecia en el cuadro 1 , en el período 1960-1973 el sector minero generaba más del 80\% de las exportaciones totales de Chile. Sin embargo, en las décadas de 1970 y 1980 esa participación disminuyó a $66,8 \%$ y $55,4 \%$, respectivamente, y en la de 1990 llegó a menos del 50\%. Por otro lado, los sectores vinculados a la explotación de recursos naturales han incrementado fuertemente sus exportaciones. Entre el período previo a la apertura y finales de la década de 1990 el sector agrícola aumentó su participación en las exportaciones chilenas de $3,2 \%$ a $10,1 \%$, el sector pesquero de $1,8 \%$ a $3,9 \%$ y el sector forestal de $1,9 \%$

\footnotetext{
5 Álvarez y Lemus (2001) encuentran que el proceso de diversificación de las exportaciones ha tenido un ritmo más lento a partir de la segunda mitad de la década de 1990, lo que sería consistente con la apreciación cambiaria del período.
} 
CUADRO 1

Chile: Exportaciones por sector, algunos períodos

(Porcentajes)

\begin{tabular}{lcccc}
\hline Período & Minería & Agricultura & Pesca & Forestal \\
\hline $1960-1973$ & 86,5 & 3,2 & 1,8 & 1,9 \\
$1974-1980$ & 66,8 & 5,5 & 4,0 & 7,3 \\
$1981-1990$ & 55,4 & 10,2 & 7,4 & 7,1 \\
$1991-1999$ & 45,9 & 10,1 & 3,9 & 32,3 \\
\hline
\end{tabular}

Fuente: Álvarez y Lemus (2001).

a 7,9\%. Pero el incremento mayor fue el experimentado por la industria manufacturera, cuya participación en las exportaciones totales aumentó sostenidamente de $10,1 \%$ a $41,9 \%$.

\section{Desempeño exportador reciente}

Si consideramos sólo la década de 1990, se aprecian algunas características adicionales del proceso de diversificación de las exportaciones chilenas. En general, la economía ha sido relativamente exitosa en las tareas de exportar nuevos productos, abrir nuevos mercados e incorporar nuevas empresas al proceso exportador. Entre 1990 y 2002, el número de productos exportados aumentó de 2.300 a 3.750, el de mercados de destino se elevó de 129 a 158 y el de empresas exportadoras de 4.100 a 6.188 (cuadro 2). Estos indicadores de desempeño exportador son sustancialmente mejores que los de décadas anteriores. ${ }^{6}$

A pesar de la positiva evolución del desempeño exportador chileno, la economía aún presenta una alta concentración de exportaciones en pocos productos, pocos mercados y, sobre todo, en un número reducido de empresas. Como se aprecia en el cuadro 3, son sólo 25 empresas las que generan casi la mitad de las exportaciones chilenas. Si agregamos las firmas que exportan entre 10 y 100 millones de dólares, menos del $4 \%$ de las empresas concentra más del $80 \%$ de las exportaciones totales.

La alta concentración de las exportaciones en un número reducido de productos se puede apreciar en el cuadro 4. A pesar de que en 2002 el país exportó más de 3.800 productos, sólo en los diez principales productos exportados se concentró el $51,1 \%$ de las exportaciones nacionales.

No obstante, la evolución fue positiva respecto a décadas anteriores. Como se aprecia en el cuadro 5, en

\footnotetext{
${ }^{6}$ Al respecto, véase Ffrench-Davis y Sáez (1995).
}

CUADRO 2

Chile: Indicadores de desempeño exportador, 1990-2002

\begin{tabular}{lrrrr}
\hline Indicador & 1990 & 1994 & 1998 & 2002 \\
\hline Empresas & 4100 & 5844 & 5847 & 6118 \\
Productos & 2300 & 3622 & 3828 & 3750 \\
Mercados & 129 & 141 & 172 & 158 \\
\hline
\end{tabular}

Fuente: DIRECON (1999) y ProChile (2002).

1970 los diez principales productos de exportación representaban casi un $90 \%$ de las exportaciones chilenas. En 1980, esa participación se había reducido a $70 \%$, y en 1994 a $59,8 \%$.

En el cuadro 6 se aprecia que, a pesar de que se han ido abriendo nuevos mercados y en la actualidad se exporta a más de 150 países, en sólo cinco de ellos se concentra alrededor del $50 \%$ de las exportaciones chilenas.

La comparación histórica muestra que la concentración de las exportaciones en un número pequeño de mercados no ha cambiado significativamente a través del tiempo. Como se aprecia en el cuadro 7, durante el período de "economía cerrada" (1960-1973), los cinco principales países de destino de las exportaciones chilenas recibían 51,6\% de ellas. En el período 1991-1999, la participación de estos cinco mercados promedió 48,9\%.

\section{Entrada y salida de empresas exportadoras}

A pesar del significativo incremento del número de empresas exportadoras, existe un fenómeno preocupante que constituye el principal motivo de esta investigación. Este fenómeno es la poca persistencia de las empresas exportadoras en los mercados internacionales. En efecto, hay evidencia de que casi un tercio de esas empresas no logra permanecer exportando más de un año. Una proporción similar corresponde a firmas 
CUADRO 3

Chile: Número de empresas y participación en las exportaciones, 2002

\begin{tabular}{|c|c|c|c|c|}
\hline Monto exportado (dólares) & $\mathrm{N}^{\mathrm{o}}$ de empresas & $\%$ de empresas & $\begin{array}{c}\text { Exportaciones } \\
\text { (millones de dólares) }\end{array}$ & $\begin{array}{c}\% \text { de las } \\
\text { exportaciones }\end{array}$ \\
\hline Menos de 100000 & 3656 & 59,8 & 78,2 & 0,4 \\
\hline Entre 100000 y 1 millón & 1483 & 24,2 & 540,9 & 3,1 \\
\hline Entre 1 y 10 millones & 757 & 12,4 & 2474,8 & 14,2 \\
\hline Entre 10 y 100 millones & 197 & 3,2 & 5773,7 & 33,1 \\
\hline Más de 100 millones & 25 & 0,4 & 8562,6 & 49,1 \\
\hline
\end{tabular}

Fuente: ProChile (2002).

CUADRO 4

Chile: Principales productos exportados, 2002

\begin{tabular}{lcr}
\hline Productos & $\begin{array}{c}\text { Miles de millones } \\
\text { de dólares }\end{array}$ & $\%$ \\
\hline Cátodos de cobre & 4095,3 & 23,5 \\
Minerales de cobre y sus concentrados & 1734,3 & 9,9 \\
Uvas frescas & 543,6 & 3,1 \\
Celulosa de coníferas, semiblanqueada & & \\
$\quad$ o blanqueada & 497,9 & 2,9 \\
Vinos con denominación de origen & 471,8 & 2,7 \\
Tablas aserradas de pino insigne & 318,3 & 2,2 \\
Harina de pescado & 318,3 & 1,8 \\
Filetes y carnes de salmón, frescos & & \\
$\quad$ o refrigerados & 300,8 & 1,7 \\
Metanol & 300,4 & 1,7 \\
Cobre para el afino & 253,7 & 1,5 \\
& & \\
Total & 8554,9 & 51,1 \\
\hline
\end{tabular}

Fuente: ProChile (2002).

CUADRO 5

Chile: Participación de los 10 principales productos exportados, algunos años

\begin{tabular}{ll}
\hline Año & $\%$ \\
\hline 1970 & 89,8 \\
1980 & 70,0 \\
1985 & 70,6 \\
1990 & 67,5 \\
1992 & 64,2 \\
1993 & 59,5 \\
1994 & 59,8 \\
\hline
\end{tabular}

Fuente: Ffrench-Davis y Sáez (1995).

que entran a los mercados internacionales sin haber exportado el año anterior. Como se aprecia en el cuadro 8, durante el período 1995-1999 sólo alrededor del $65 \%$ de las aproximadamente 6.000 empresas exportadoras estaba constituido por firmas que pueden calificarse de exportadoras constantes por más de un año.
CUADRO 6

Chile: Principales mercados de destino, 2002

\begin{tabular}{lc}
\hline País & $\begin{array}{c}\text { Participación en las exportaciones } \\
(\%)\end{array}$ \\
\hline Estados Unidos & 19,8 \\
Japón & 10,8 \\
China & 7,0 \\
México & 5,2 \\
Reino Unido & 4,9 \\
Total & 47,7 \\
\hline
\end{tabular}

Fuente:ProChile (2002).

CUADRO 7

Chile: Participación por mercados de destino principales

\begin{tabular}{lccccc}
\hline Período & 5 países & 10 países & 15 países & 20 países & 25 países \\
\hline $1960-1973$ & 51,6 & 78,2 & 90,8 & 93,3 & 97,7 \\
$1974-1980$ & 47,0 & 70,1 & 81,3 & 89,5 & 92,1 \\
$1981-1990$ & 47,7 & 68,0 & 81,6 & 87,8 & 89,9 \\
$1991-1999$ & 48,9 & 66,3 & 81,3 & 87,3 & 89,9 \\
\hline
\end{tabular}

Fuente: Álvarez y Lemus (2001).

Los antecedentes presentados en esta sección ponen de manifiesto la existencia de un fenómeno interesante de estudiar. Aunque ha habido una positiva evolución de los indicadores de diversificación de las exportaciones chilenas y existe relativo consenso en que el sector exportador está hoy significativamente más diversificado que en décadas precedentes, persisten algunos problemas importantes, como la alta rotación de las empresas exportadoras. Aun cuando existen estudios relativos a este fenómeno en otras economías del mundo, en Chile se carece de evidencia empírica al respecto.

Una mayor consolidación de las empresas exportadoras podría ayudar a reducir la concentración de las exportaciones chilenas, a través de la introducción de 
Chile: Dinámica de la entrada y salida de empresas, 1995-2002

(Número de empresas y porcentajes)

\begin{tabular}{|c|c|c|c|c|c|c|c|}
\hline \multirow{2}{*}{$\begin{array}{l}\text { Año } \\
1995\end{array}$} & \multicolumn{2}{|c|}{ Entrantes } & \multicolumn{2}{|c|}{ Salientes } & \multicolumn{2}{|c|}{ Constantes } & \multirow{2}{*}{$\begin{array}{c}\text { Totales } \\
5813\end{array}$} \\
\hline & 2066 & 35,5 & 2093 & 36,0 & 3748 & 64,5 & \\
\hline 1996 & 2094 & 36,1 & 2102 & 36,2 & 3711 & 63,9 & 5805 \\
\hline 1997 & 1988 & 34,5 & 2026 & 35,1 & 3779 & 65,5 & 5767 \\
\hline 1998 & 2007 & 34,3 & 1927 & 33,0 & 3840 & 65,7 & 5847 \\
\hline 1999 & 2121 & 35,2 & 1946 & 32,3 & 3901 & 64,8 & 6022 \\
\hline 2000 & 1826 & 32,2 & 2182 & 38,5 & 3840 & 0,68 & 5666 \\
\hline 2001 & 2174 & 36,2 & 1831 & 30,5 & 3835 & 0,64 & 6009 \\
\hline 2002 & 2164 & 35,4 & 2055 & 33,6 & 3954 & 0,65 & 6118 \\
\hline
\end{tabular}

Fuente: ProChile (2000) y (2002).

nuevos productos y la apertura de nuevos mercados. En efecto, como se muestra en la sección siguiente, las firmas que exportan permanentemente venden más productos en los mercados internacionales y llegan a un número mayor de mercados. Así, la conexión entre la diversificación de las exportaciones y la consolidación de las empresas está dada por el hecho de que las empresas que exportan permanentemente están en mejores condiciones de incorporar nuevos productos y mercados a la canasta exportadora chilena.

\section{III}

\section{Características de las empresas exportadoras}

\section{Fuente de los datos}

Para estudiar las características de las empresas y su estatus exportador se cuenta con dos fuentes de información complementarias. La primera corresponde a las estadísticas de exportaciones del Banco Central de Chile, con datos provenientes del Servicio Nacional de Aduanas. Esta base de datos cuenta con información de todas las empresas exportadoras en el período 19911999 que cubre variables de desempeño exportador como el valor de las exportaciones (medidas en dólares), el número de mercados de destino (o países a los que se exporta) y el número de productos exportados. La segunda fuente es la base de datos de la Encuesta Nacional Industrial Anual (ENIA) para el período 19901996, que contiene información sobre diversas características de las firmas, como empleo, valor agregado, ventas e inversión, que pueden ser importantes para explicar su desempeño exportador.

Una de las deficiencias que tiene la primera fuente de información es que las empresas importadoras que devuelven mercancías quedan registradas como expor- tadoras en los datos que se originan en el Servicio Nacional de Aduanas; en tal caso, habría una sobreestimación del número de empresas exportadoras $\mathrm{y}$, en mayor medida, del número de firmas que exportan esporádicamente. Además, carece de información sobre variables que no sean aquellas relativas al comportamiento exportador de las firmas.

Una falencia de la ENIA es que sólo cuenta con información acerca del valor de las exportaciones y no de los mercados de destino ni de los productos exportados. Además, esta encuesta sólo se aplica a empresas manufactureras.

Por tales motivos, el análisis que se muestra a continuación utiliza ambas fuentes de información de manera complementaria.

\section{Algunos hechos estilizados}

Con la información proveniente del Banco Central es posible analizar las principales características de la dinámica de la entrada y salida de firmas exportadoras en el período 1991-1999, identificando los principales hechos estilizados del fenómeno. 
Para ello, se dividen las empresas exportadoras en dos grupos: las exportadoras permanentes, que se definen como aquellas empresas que mantienen su actividad exportadora todos los años bajo estudio, y las exportadoras esporádicas, que son aquellas que una vez que entran a los mercados internacionales, salen en algún año posterior.

\section{a) Un porcentaje mayoritario de empresas no expor- ta permanentemente}

Uno de los primeros hechos estilizados es que la tasa de entrada y salida de firmas es relativamente alta. De hecho, durante el período 1991-1999 sólo un 6\% de las firmas puede catalogarse entre las exportadoras permanentes y el $94 \%$ restante corresponde a empresas que han exportado esporádicamente (cuadro 9).

No obstante, a medida que se reduce el período de análisis, y por ello se relaja en cierta medida el criterio para definir las exportadoras permanentes, éstas incrementan su participación en el total de exportadoras. En definitiva, mientras más corto es el intervalo de tiempo analizado, mayor es el porcentaje de empresas a las que se puede definir como exportadoras permanentes. En efecto, si se toma el período 19961999, un 23\% de las firmas exportaron todos los años del período y un $77 \%$ lo hizo esporádicamente. Aunque el porcentaje de exportadoras esporádicas se reduce, no deja de representar una magnitud considerable.

\section{b) La evidencia es similar a nivel sectorial}

Un segundo hecho estilizado es que a nivel sectorial también se encuentra que entre las empresas exportadoras es mayoritario el porcentaje de las que exportan esporádicamente.

No obstante, cabe destacar algunas diferencias. Como se ve en el cuadro 10, en el período 1991-1999 aproximadamente el $18 \%$ de las empresas exportadoras pertenecientes al sector cobre y hierro pueden calificarse de exportadoras permanentes. Otros sectores en los cuales las exportadoras permanentes tienen una participación mayor son los de metales no ferrosos $(14,2 \%)$, petróleo y derivados $(12,9 \%)$, productos plásticos $(11,5 \%)$, papel y celulosa $(11,1 \%)$ y otros minerales y piedras $(11,0 \%)$. En cambio, en sectores como los de servicios, muebles de madera, material de transporte y carbón, las empresas exportadoras son fundamentalmente del tipo esporádico.

\section{c) Existen diferencias al inicio del proceso expor- tador de las empresas}

Una tercera característica importante es que las firmas que se definen como exportadoras permanen-
CUADRO 9

Chile: Empresas exportadoras por categoría y por períodos

\begin{tabular}{|c|c|c|c|c|c|}
\hline \multirow[t]{2}{*}{ Período } & \multicolumn{2}{|c|}{ Esporádicas } & \multicolumn{2}{|c|}{ Permanentes } & \multirow{2}{*}{$\frac{\text { Total }}{\mathrm{N}^{\circ}}$} \\
\hline & $\mathrm{N}^{\circ}$ & $\%$ & $\mathrm{~N}^{\circ}$ & $\%$ & \\
\hline 1991-1999 & 16255 & 94,1 & 1015 & 5,9 & 17270 \\
\hline 1992-1999 & 14597 & 92,4 & 1198 & 7,6 & 15795 \\
\hline 1993-1999 & 13016 & 90,2 & 1420 & 9,8 & 14436 \\
\hline 1994-1999 & 11487 & 87,3 & 1667 & 12,7 & 13154 \\
\hline 1995-1999 & 9739 & 83,3 & 1949 & 16,7 & 11688 \\
\hline 1996-1999 & 7897 & 77,3 & 2326 & 22,7 & 10223 \\
\hline
\end{tabular}

Fuente: Elaboración propia sobre la base de estadísticas del Banco Central de Chile.

CUADRO 10

Chile: Empresas exportadoras por categoría y sector, 1991-1999

\begin{tabular}{|c|c|c|c|}
\hline Sector ${ }^{a}$ & Descripción & $\begin{array}{l}\text { Esporádicas } \\
(\%)\end{array}$ & $\begin{array}{c}\text { Permanentes } \\
(\%)\end{array}$ \\
\hline $111-130$ & Agricultura y pesca & 92,3 & 7,7 \\
\hline 220 & Cobre y hierro & 82,1 & 18,0 \\
\hline 212 & Carbón & 100,0 & 0,0 \\
\hline $230-240$ & Otros minerales y piedras & 89,0 & 11,0 \\
\hline $311-313$ & Alimentos, bebidas y tabaco & 90,1 & 9,9 \\
\hline 321 & Textiles & 94,3 & 5,7 \\
\hline 322 & Vestuario & 97,0 & 3,0 \\
\hline 323 & Cuero y calzado & 93,7 & 6,3 \\
\hline 331 & Maderas, exc. muebles & 94,0 & 6,0 \\
\hline 332 & Muebles, exc. metálicos & 98,7 & 1,3 \\
\hline 341 & Papel y celulosa & 88,9 & 11,1 \\
\hline 3432 & Imprenta y editoriales & 94,8 & 5,2 \\
\hline 351 & Sustancias y prod. químicos & 91,1 & 8,9 \\
\hline 352 & Petróleo y derivados & 87,1 & 12,9 \\
\hline 361 & Productos de caucho & 95,7 & 4,3 \\
\hline 362 & Productos plásticos & 88,5 & 11,5 \\
\hline 369 & Barro, loza y vidrio & 92,1 & 7,9 \\
\hline 371 & Ind. básicas de hierro y acero & 93,0 & 7,0 \\
\hline 372 & Metales no ferrosos & 85,9 & 14,2 \\
\hline 381 & Productos metálicos & 93,8 & 6,2 \\
\hline 382 & Maquinaria no eléctrica & 96,8 & 3,3 \\
\hline 383 & Maquinaria y aparatos eléctricos & 96,7 & 3,3 \\
\hline 384 & Material de transporte & 99,4 & 0,6 \\
\hline 385 & Equipo prof. y científico & 96,4 & 3,6 \\
\hline 390 & Otras industrias & 95,5 & 4,5 \\
\hline 900 & Servicios & 97,6 & 2,4 \\
\hline
\end{tabular}

Fuente: Elaboración propia sobre la base de estadísticas del Banco Central de Chile.

a Según la Clasificación Industrial Internacional Uniforme de todas las actividades económicas (CIIU).

tes presentan mejores indicadores iniciales de desempeño exportador que las empresas que exportan esporádicamente.

En el cuadro 11 se muestran los resultados de la comparación entre ambos grupos de empresas, utilizando 
CUADRO 11

Chile: Indicadores de desempeño por categoría de empresas

\begin{tabular}{|c|c|c|c|c|c|c|}
\hline Variable & Permanentes & $\begin{array}{c}\text { Esporádicas } \\
1991-1999\end{array}$ & Diferencia $^{a}$ & Permanentes & $\begin{array}{c}\text { Esporádicas } \\
\text { 1996-1999 }\end{array}$ & Diferencia $^{\mathrm{a}}$ \\
\hline Exportaciones $^{\mathrm{b}}$ & 4,6 & 1,1 & $\begin{array}{c}3,4 \\
(2,38)\end{array}$ & 4,9 & 1,4 & $\begin{array}{c}3,5 \\
(2,39)\end{array}$ \\
\hline Mercados & 4,7 & 1,8 & $\begin{array}{c}2,9 \\
(27,86)\end{array}$ & 4,6 & 1,6 & $\begin{array}{c}3,0 \\
(27,11)\end{array}$ \\
\hline Productos & 7,0 & 3,9 & $\begin{array}{c}3,1 \\
(10,02)\end{array}$ & 7,9 & 3,6 & $\begin{array}{c}4,3 \\
(12,02)\end{array}$ \\
\hline
\end{tabular}

a Las cifras entre paréntesis corresponden a estadísticos t de igualdad de medias.

b Medidas en millones de dólares corrientes.

el período completo 1991-1999 y un período más reciente, el de 1996-1999. Para ambos períodos se clasificaron las empresas exportadoras en esporádicas y permanentes y luego se realizó una prueba de diferencias de medias para tres indicadores de desempeño exportador inicial: valor de las exportaciones, mercados de destino y número de productos exportados.

En todos los indicadores analizados, el desempeño inicial de los exportadores permanentes es superior al de los exportadores esporádicos. Además, en todos los casos la diferencia de medias es estadísticamente significativa.

En el período 1991-1999, las empresas exportadoras permanentes exportaron en el año inicial (1991), como promedio, 3,4 millones de dólares más que las que no se consolidaron en los mercados internacionales. Además, tenían casi tres mercados más de destino y exportaban tres productos adicionales. Los resultados son similares si el período de comparación es 19961999, salvo para los productos exportados, en los cuales la diferencia entre exportadores permanentes y esporádicos se incrementa en aproximadamente tres a cuatro productos en ambos períodos.

\section{d) El efecto mercado parece no ser importante}

El hecho de ser un exportador permanente o un exportador esporádico podría tener relación con el mercado donde las empresas envían sus productos. Este efecto mercado ocurriría si en algunos países se utilizaran en mayor medida políticas comerciales proteccionistas que perjudicaran la participación de empresas chilenas en los mercados internacionales.

Para analizar este fenómeno, se ha clasificado a las empresas de acuerdo a los principales mercados de destino de las exportaciones chilenas. Aunque una empresa puede exportar a varios países, en este caso la clasificación se ha hecho de acuerdo con el princi- pal país de destino de sus exportaciones. ${ }^{7}$ Según el cuadro 12, pareciera no existir un fenómeno como el descrito. Salvo en Japón, donde el porcentaje de firmas exportadoras permanentes es más alto $(17,2 \%)$, en el resto de los socios comerciales de Chile existe una distribución similar entre ambos grupos de empresas.

Se podría argumentar que un porcentaje importante de los exportadores esporádicos surge por el denominado "intercambio fronterizo", un tipo de comercio de carácter transitorio y de pequeña magnitud que depende de circunstancias fortuitas, y que no está dado necesariamente por estrategias empresariales y/o ventajas comparativas. ${ }^{8}$ Si esto fuera cierto, la probabilidad de ser exportador esporádico sería mayor si la empresa exportara mayoritariamente a los países fronterizos o más cercanos. Efectivamente, el porcentaje de exportadores chilenos que no se consolidan es mayor entre los que venden al Mercosur y al resto de los países latinoamericanos, y las diferencias son estadísticamente significativas, aunque de poca magnitud. ${ }^{9}$

\section{e) La evidencia es similar en la industria manufac- turera}

Utilizando información de la ENIA elaborada por el Instituto Nacional de Estadísticas (INE), para todos los años comprendidos entre 1990 y 1996, se puede agregar un tercer grupo de empresas, las no exportadoras.

\footnotetext{
${ }^{7}$ Por ejemplo, una empresa que exporta mayoritariamente a Argentina queda clasificada como exportadora al Mercosur.

${ }^{8}$ Agradezco este punto a Dominique Hachette.

${ }^{9}$ Con el fin de explorar este fenómeno, se estimó un modelo probit con variable dependiente dicotómica igual a 1 para los exportadores permanentes, y 0 para el resto (exportadores esporádicos). Como variable explicativa se incluyó una variable categórica por mercado. Los parámetros para el Mercosur y el resto de los países latinoamericanos resultaron negativos y significativos, pero señalaron una diferencia pequeña de sólo 2 a 3 puntos porcentuales.
} 
CUADRO 12

Mundo: Distribución de empresas exportadoras esporádicas y permanentes en los socios comerciales de Chile, 1991-1999

\begin{tabular}{|c|c|c|c|c|c|}
\hline \multirow{2}{*}{$\frac{\text { Socio comercial }}{\text { Unión Europea }}$} & \multicolumn{2}{|c|}{ Esporádicas } & \multicolumn{2}{|c|}{ Permanentes } & \multirow{2}{*}{$\begin{array}{l}\text { Total } \\
2527\end{array}$} \\
\hline & 2357 & 93,3 & 170 & 6,7 & \\
\hline Tratado de Libre Comercio de América del Norte & 3009 & 92,6 & 241 & 7,4 & 3250 \\
\hline Mercosur & 4668 & 95,9 & 202 & 4,1 & 4870 \\
\hline Resto de América Latina & 4905 & 95,3 & 240 & 4,7 & 5145 \\
\hline Japón & 379 & 82,8 & 79 & 17,2 & 458 \\
\hline Otros de Asia ${ }^{a}$ & 270 & 93,4 & 19 & 6,6 & 289 \\
\hline Total & 16255 & 94,1 & 1015 & 5,9 & 17270 \\
\hline
\end{tabular}

Fuente: Elaboración propia en base a estadísticas del Banco Central de Chile.

a Incluye China, Filipinas, Indonesia, Malasia, República de Corea, Singapur, Tailandia y la provincia china de Taiwán.

CUADRO 13

Chile: Clasificación de las empresas según su estatus exportador, por sectores

(Porcentajes)

\begin{tabular}{|c|c|c|c|c|}
\hline Sector & $\begin{array}{c}\text { Empresas } \\
\text { no exportadoras }\end{array}$ & $\begin{array}{l}\text { Exportadoras } \\
\text { esporádicas }\end{array}$ & $\begin{array}{l}\text { Exportadoras } \\
\text { permanentes }\end{array}$ & $\begin{array}{c}\text { Exportadoras } \\
\text { esporádicas sobre el } \\
\text { total de exportadoras }\end{array}$ \\
\hline Alimentos & 77,1 & 17,4 & 5,5 & 76,0 \\
\hline Alimentos forrajeros & 59,6 & 30,3 & 10,1 & 75,0 \\
\hline Bebidas & 54,5 & 30,4 & 15,2 & 66,7 \\
\hline Tabaco & 20,0 & 80,0 & 0,0 & 100,0 \\
\hline Textiles & 71,6 & 22,7 & 5,7 & 79,9 \\
\hline Vestuario & 79,7 & 18,6 & 1,7 & 91,7 \\
\hline Productos de cuero & 63,5 & 32,4 & 4,1 & 88,9 \\
\hline Calzado & 70,3 & 25,2 & 4,5 & 84,8 \\
\hline Maderas, exc. muebles & 70,6 & 23,9 & 5,5 & 81,2 \\
\hline Muebles, exc. metálicos & 85,0 & 12,4 & 2,6 & 82,9 \\
\hline Papel y celulosa & 48,5 & 39,8 & 11,7 & 77,4 \\
\hline Imprenta y editoriales & 81,3 & 17,3 & 1,4 & 92,3 \\
\hline Productos químicos & 48,1 & 36,8 & 15,1 & 70,9 \\
\hline Otros prod. químicos & 49,8 & 37,0 & 13,2 & 73,6 \\
\hline Refinerías de petróleo & 0,0 & 0,0 & 100,0 & 0,0 \\
\hline Derivados del petróleo & 42,9 & 42,9 & 14,3 & 75,0 \\
\hline Productos de caucho & 71,1 & 18,4 & 10,5 & 63,6 \\
\hline Productos plásticos & 67,3 & 28,3 & 4,5 & 86,4 \\
\hline Cerámica & 70,0 & 16,7 & 13,3 & 55,6 \\
\hline Vidrio & 47,6 & 23,8 & 28,6 & 45,5 \\
\hline Otros min. no metálicos & 89,0 & 8,6 & 2,4 & 78,3 \\
\hline Ind. básicas hierro y acero & 64,6 & 20,8 & 14,6 & 58,8 \\
\hline Metales no ferrosos & 37,5 & 41,1 & 21,4 & 65,7 \\
\hline Productos metálicos & 80,4 & 16,8 & 2,8 & 85,7 \\
\hline Maquinaria no eléctrica & 77,9 & 18,5 & 3,7 & 83,3 \\
\hline Maquinaria eléctrica & 52,9 & 38,8 & 8,2 & 82,5 \\
\hline Material de transporte & 74,6 & 23,1 & 2,4 & 90,7 \\
\hline Equipo prof. y científico & 54,5 & 36,4 & 9,1 & 80,0 \\
\hline Total & 73,0 & 21,5 & 5,6 & 79,4 \\
\hline
\end{tabular}

Fuente. Elaboración propia sobre la base de información de la ENIA.

En el cuadro 13 se muestra la distribución de las empresas manufactureras según los tres tipos de estatus exportador. Las empresas del sector industrial son mayoritariamente no exportadoras (73\%); un 21,5\% corresponde a empresas que exportan esporádicamente, y sólo un $5,6 \%$ se puede calificar de exportadoras 
permanentes. Si consideramos sólo las empresas exportadoras, casi un $80 \%$ corresponde a empresas que no exportan permanentemente.

Este resultado para la industria manufacturera es consistente con lo encontrado para el universo de empresas exportadoras (véase más atrás el cuadro 9). Con datos de la ENIA, que no tienen el problema de incluir empresas importadoras catalogadas como exportadoras en la información provista por el Banco Central, sigue siendo válido sostener que un porcentaje mayoritario de firmas exportadoras corresponde a aquéllas que no son capaces de mantenerse en los mercados internacionales. Es decir, el fenómeno de alta presencia de empresas exportadoras es independiente de la base de datos utilizada para la definición de ambos grupos de empresas y del período que se habrá de considerar.

Analizando la distribución de empresas por sectores puede inferirse que, salvo algunas excepciones, el porcentaje mayoritario de empresas exportadoras corresponde a las de actividad exportadora esporádica.
Existe la posibilidad de que se esté exagerando la importancia relativa de los exportadores esporádicos, pues algunos son empresas que no sólo han dejado de exportar, sino también que han salido del mercado. ${ }^{10}$ No obstante, si sólo consideramos las empresas que han permanecido en el mercado los siete años estudiados, efectivamente se reduce la importancia relativa de las empresas exportadoras que no se consolidan, aunque ésta sigue siendo más bien alta.

Tomando las empresas que han permanecido en el mercado el período completo, en comparación con la muestra total, se observa que el porcentaje de no exportadoras se reduce de $73,0 \%$ a $64,2 \%$, el de exportadoras esporádicas aumenta de $21,5 \%$ a $23,1 \%$ y el de exportadoras permanentes se eleva a $12,7 \%$. Esto significa que, aun considerando el grupo de firmas que sobreviven todo el período en estudio, la mayor parte de las empresas que exportan sólo lo hace esporádicamente. La principal razón de este predominio de las exportadoras esporádicas es que las firmas que han salido del mercado han sido primordialmente las que no exportaban.

\section{IV}

\section{Desempeño comparativo por grupos}

\section{de empresas}

Diversos trabajos empíricos han confirmado que las empresas exportadoras muestran mejores características que las que venden solamente en el mercado interno. En general, las firmas exportadoras son más grandes, más productivas, hacen uso más intensivo de capital y tecnología, y pagan salarios mayores. ${ }^{11}$

Para evaluar si esto es efectivo en el caso chileno, se estudian las características de las empresas manufactureras en los tres grupos definidos anteriormente. En el gráfico 2 se muestran algunos indicadores de tamaño, productividad y densidad de capital físico para empresas no exportadoras, exportadoras esporádicas y exportadoras permanentes.

\footnotetext{
10 Agradezco a Bernardita Escobar por este punto.

11 Varios estudios documentan este fenómeno en empresas: por ejemplo, Bernard y Jensen (1999) para Estados Unidos, Bernard y Wagner (2001) para Alemania, Isgut (2001) para Colombia, y Baldwin y Gu (2003) para Canadá.
}

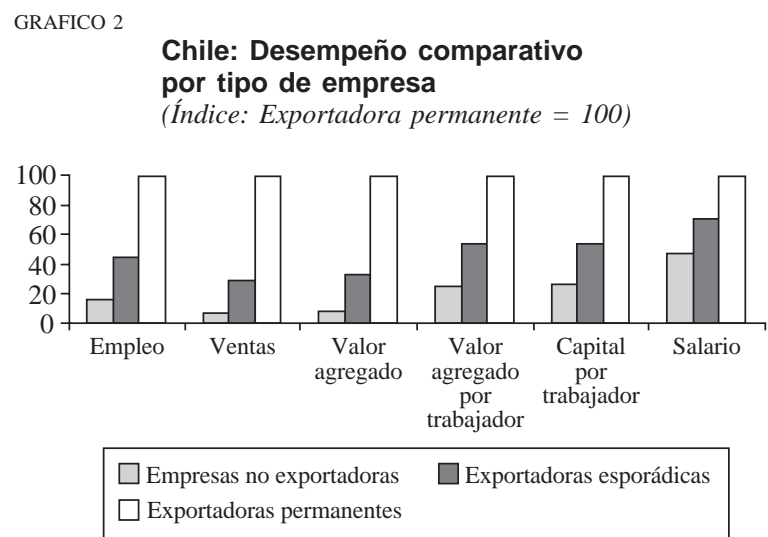

Fuente: Elaboración propia con datos de la ENIA.

Los indicadores de tamaño, como ventas y empleo, muestran que las firmas que exportan permanentemente son "más grandes" que aquellas que no exportan o que exportan esporádicamente. Las empresas no 
exportadoras y las exportadoras esporádicas tienen alrededor de $20 \%$ y $40 \%$, respectivamente, del tamaño de las exportadoras permanentes.

En términos de productividad laboral, medida por el valor agregado por trabajador y el salario promedio, las diferencias entre grupos de empresas son también bastante amplias. Las que exportan permanentemente son "más productivas" que las que exportan esporádicamente, y aún más que las no exportadoras. Estas diferencias de productividad podrían deberse a las disparidades en la intensidad con que se hace uso de capital. Se puede apreciar en el gráfico 2 que las exportadoras permanentes hacen uso más intensivo de capital que las exportadoras esporádicas y que las no exportadoras.

Siguiendo a Bernard y Jensen (1999), ${ }^{12}$ para verificar si las exportadoras permanentes muestran un desempeño significativamente superior al resto utilizando un grupo más amplio de indicadores, se procede a estimar lo siguiente:

$$
\mathrm{Y}_{\mathrm{it}}=\alpha_{0}+\alpha_{1} \mathrm{D}_{\mathrm{i}}^{\mathrm{ES}}+\alpha_{2} \mathrm{D}_{\mathrm{i}}^{\mathrm{p}}+\alpha_{3} \mathrm{Z}_{\mathrm{it}}+\varepsilon_{\mathrm{it}}
$$

donde $Y$ es alguna variable característica de la empresa (medida en logaritmo), $D^{E S}$ es una variable categórica si la empresa exporta esporádicamente, $D^{p}$ es una variable categórica si la empresa exporta permanentemente, $\mathrm{Z}$ es un vector de variables de control, como año y sector productivo, $i$ denota la empresa y $t$ el año. $\mathrm{Al}$ incluir una variable categórica por sector, se procede a comparar las empresas, considerando las diferencias asociadas al sector al que pertenecen. Es decir, dentro de un mismo sector se analiza si las empresas exportadoras muestran mejores características que las no exportadoras. Los sectores corresponden a la Clasificación Industrial Internacional Uniforme de todas las actividades económicas (CIIU), a tres dígitos.

Si $\alpha_{l}$ es positivo, podemos inferir que las empresas exportadoras esporádicas muestran un mejor desempeño que las no exportadoras. Análogamente, si $\alpha_{2}$ es positivo y mayor que $\alpha_{1}$, entonces las empresas que exportan permanentemente tienen características superiores a las exportadoras esporádicas y a las no exportadoras.

Los resultados de las estimaciones se pueden apreciar en el cuadro 14. En la segunda columna de este cuadro se muestra el parámetro asociado a la va-

\footnotetext{
12 A diferencia del presente trabajo, estos autores aplican la metodología a sólo dos grupos de empresas, las exportadoras y las no exportadoras.
}

riable que identifica a las empresas esporádicas $\left(\alpha_{1}\right)$, y en la tercera, el parámetro de la variable que identifica a las exportadoras permanentes $\left(\alpha_{2}\right)$. En la última columna se presenta la diferencia entre ambos parámetros $\left(\alpha_{2}-\alpha_{1}\right)$. Esta diferencia puede interpretarse como una especie de "premio" por avanzar en la consolidación exportadora, pasando de la exportación esporádica a la permanente.

De acuerdo con los resultados de las estimaciones, el parámetro $\alpha_{1}$ es siempre positivo y significativo, lo que permite inferir que el desempeño de las empresas exportadoras esporádicas es superior al de las no exportadoras. En términos de tamaño, las primeras utilizan casi el doble de trabajadores y generan aproximadamente dos a tres veces más valor agregado, valor bruto de producción y ventas. Su acervo de capital es

CUADRO 14 Chile: Comparación de características por
grupos de empresas (Promedio 1990-1996)

\begin{tabular}{|c|c|c|c|}
\hline Variable & $\begin{array}{l}\text { Exportadoras } \\
\text { esporádicas }\end{array}$ & $\begin{array}{l}\text { Exportadoras } \\
\text { permanentes }\end{array}$ & Diferencia \\
\hline Empleo total & $\begin{array}{c}0,92 \\
(79,07)\end{array}$ & $\begin{array}{c}1,78 \\
(99,13)\end{array}$ & 0,86 \\
\hline Valor agregado & $\begin{array}{c}1,38 \\
(77,96)\end{array}$ & $\begin{array}{c}2,70 \\
(97,97)\end{array}$ & 1,32 \\
\hline Ventas & $\begin{array}{c}1,37 \\
(78,78)\end{array}$ & $\begin{array}{c}2,65 \\
(99,17)\end{array}$ & 1,28 \\
\hline Valor bruto & $\begin{array}{c}1,33 \\
(7,65)\end{array}$ & $\begin{array}{c}2,57 \\
(98,18)\end{array}$ & 1,24 \\
\hline Acervo de capital & $\begin{array}{c}1,59 \\
(70,14)\end{array}$ & $\begin{array}{c}3,01 \\
(90,97)\end{array}$ & 1,42 \\
\hline Valor agregado por trabajador & $\begin{array}{c}0,47 \\
(40,51)\end{array}$ & $\begin{array}{c}0,92 \\
(51,15)\end{array}$ & 0,47 \\
\hline Valor bruto por trabajador & $\begin{array}{c}0,41 \\
(38,38)\end{array}$ & $\begin{array}{c}0,78 \\
(47,44)\end{array}$ & 0,37 \\
\hline Ventas por trabajador & $\begin{array}{c}0,43 \\
(37,66)\end{array}$ & $\begin{array}{c}0,85 \\
(47,89)\end{array}$ & 0,42 \\
\hline Salario promedio & $\begin{array}{c}0,32 \\
(45,80)\end{array}$ & $\begin{array}{c}0,62 \\
(58,17)\end{array}$ & 0,30 \\
\hline Salario promedio empleados & $\begin{array}{c}0,45 \\
(50,30)\end{array}$ & $\begin{array}{c}0,82 \\
(59,46)\end{array}$ & 0,37 \\
\hline Salario promedio obreros & $\begin{array}{c}0,20 \\
(30,53)\end{array}$ & $\begin{array}{c}0,43 \\
(42,15)\end{array}$ & 0,23 \\
\hline Capital por trabajador & $\begin{array}{c}0,69 \\
(42,54)\end{array}$ & $\begin{array}{c}1,24 \\
(50,04)\end{array}$ & 0,55 \\
\hline $\begin{array}{l}\text { Participación de calificados } \\
\text { en el empleo total }\end{array}$ & $\begin{array}{c}0,09 \\
(9,12)\end{array}$ & $\begin{array}{c}0,20 \\
(13,58)\end{array}$ & 0,11 \\
\hline Gasto en licencias de venta & $\begin{array}{c}0,06 \\
(2,11)\end{array}$ & $\begin{array}{c}0,11 \\
(2,48)\end{array}$ & $0,05^{\mathrm{c}}$ \\
\hline
\end{tabular}

a Las cifras entre paréntesis corresponden a estadísticos t.

b En el cuadro no se incluyen los parámetros del término constante ni las variables categóricas por sectores productivos.

c No significativa al $5 \%$. 
2,6 veces el de una firma no exportadora. Su productividad laboral, medida a través del valor agregado por trabajador, el valor bruto por trabajador o las ventas por trabajador, es $40 \%$ a $50 \%$ mayor que la productividad de las empresas no exportadoras, lo que, como era de esperar, les permite pagar salarios un $32 \%$ más altos en promedio ( $45 \%$ en el caso de los empleados y $20 \%$ en el de los obreros). El hecho de que la productividad de las exportadoras esporádicas sea más alta que la de las empresas no exportadoras podría deberse a

CUADRO 15

\section{Chile: Comparación de características iniciales por grupos de empresas exportadoras ${ }^{\mathrm{ab}}$}

\begin{tabular}{|c|c|c|c|}
\hline Variable & $\begin{array}{l}\text { Exportadoras } \\
\text { esporádicas }\end{array}$ & $\begin{array}{l}\text { Exportadoras } \\
\text { permanentes }\end{array}$ & Diferencia \\
\hline Empleo total & $\begin{array}{c}0,89 \\
(26,8)\end{array}$ & $\begin{array}{c}1,73 \\
(35,9)\end{array}$ & 0,84 \\
\hline Valor agregado & $\begin{array}{c}1,41 \\
(27,5)\end{array}$ & $\begin{array}{c}2,68 \\
(36,0)\end{array}$ & 1,27 \\
\hline Ventas & $\begin{array}{c}1,37 \\
(28,5)\end{array}$ & $\begin{array}{c}2,59 \\
(37,0)\end{array}$ & 1,22 \\
\hline Valor bruto & $\begin{array}{c}1,30 \\
(27,6)\end{array}$ & $\begin{array}{c}2,48 \\
(36,0)\end{array}$ & 1,18 \\
\hline Acervo de capital & $\begin{array}{l}1,53 \\
(25,3)\end{array}$ & $\begin{array}{c}2,91 \\
(33,1)\end{array}$ & 1,38 \\
\hline Valor agregado por trabajador & $\begin{array}{c}0,52 \\
(15,5)\end{array}$ & $\begin{array}{c}0,95 \\
(19,4)\end{array}$ & 0,43 \\
\hline Valor bruto por trabajador & $\begin{array}{c}0,42 \\
(14,1)\end{array}$ & $\begin{array}{c}0,75 \\
(17,3)\end{array}$ & 0,33 \\
\hline Ventas por trabajador & $\begin{array}{c}0,47 \\
(14,6)\end{array}$ & $\begin{array}{c}0,84 \\
(18,0)\end{array}$ & 0,37 \\
\hline Salario promedio & $\begin{array}{c}0,34 \\
(17,2)\end{array}$ & $\begin{array}{c}0,64 \\
(22,4)\end{array}$ & 0,30 \\
\hline Salario promedio empleados & $\begin{array}{c}0,50 \\
(19,1)\end{array}$ & $\begin{array}{c}0,90 \\
(23,6)\end{array}$ & 0,40 \\
\hline Salario promedio obreros & $\begin{array}{c}0,22 \\
(11,8)\end{array}$ & $\begin{array}{c}0,43 \\
(16,1)\end{array}$ & 0,21 \\
\hline Capital por trabajador & $\begin{array}{c}0,67 \\
(14,7)\end{array}$ & $\begin{array}{c}1,21 \\
(18,4)\end{array}$ & 0,54 \\
\hline $\begin{array}{l}\text { Participación de calificados } \\
\text { en empleo total }\end{array}$ & $\begin{array}{l}0,09 \\
(3,6)\end{array}$ & $\begin{array}{l}0,19 \\
(5,1)\end{array}$ & 0,10 \\
\hline Gasto en licencias de venta & $\begin{array}{l}0,05 \\
(2,1)\end{array}$ & $\begin{array}{l}0,09 \\
(2,8)\end{array}$ & $0,04^{\mathrm{c}}$ \\
\hline
\end{tabular}

a Las cifras entre paréntesis corresponden a estadísticos t.

b En el cuadro no se incluyen los parámetros del término constante ni las variables categóricas por sectores productivos.

c No significativa al $5 \%$. que las primeras cuentan con mayor capital por trabajador (69\% más), y con una mayor densidad de capital humano. La participación de los empleados (calificados) en el empleo total es un $9 \%$ más alta. Finalmente, existen también importantes diferencias en la densidad tecnológica. Las exportadoras esporádicas tienen un gasto en licencias de venta que es 0,06 puntos porcentuales mayor que el de las no exportadoras.

La comparación entre empresas exportadoras muestra que las exportadoras permanentes tienen mejores características que las exportadoras esporádicas. Las estimaciones indican que el parámetro $\alpha_{2}$ es positivo, significativo y mayor que $\alpha_{1}$. Las empresas que exportan permanentemente generan $86 \%$ más empleo y entre $120 \%$ y $130 \%$ más producción y ventas que las exportadoras esporádicas; además, son entre $40 \%$ y $50 \%$ más productivas, y pagan salarios entre $20 \%$ y $40 \%$ más altos. Su capital por trabajador es casi $60 \%$ superior y la densidad de capital humano en ellas es $11 \%$ mayor. En esta comparación no se observan disparidades estadísticamente significativas en el gasto en licencias entre exportadoras esporádicas y permanentes.

Las diferencias entre los grupos de empresas examinados, como se mostró previamente al comparar las variables relativas al desempeño exportador inicial, son también evidentes al inicio del período, y no sólo al considerar el promedio de los años considerados. En el cuadro 15 se puede apreciar que, según la mayoría de los indicadores, ya en 1990 las empresas que exportaban permanentemente tenían un mejor desempeño que las exportadoras esporádicas y que las no exportadoras. ${ }^{13}$ Estos resultados sugieren que existe una correlación positiva entre el desempeño de las firmas y su estatus exportador. Las empresas que se consolidan en los mercados internacionales son aquellas que presentan mayor productividad, tienen un tamaño superior, cuentan con más capital físico y humano, y pueden pagar salarios más altos.

\footnotetext{
${ }^{13}$ La diferencia con la estimación anterior es que no se incluye la variable categórica por año, sino que todas las variables bajo comparación están medidas en el año inicial.
} 


\section{V}

\section{Conclusiones}

El desempeño exportador chileno desde mediados del decenio de 1980 ha sido particularmente exitoso, exhibiendo un fuerte crecimiento de las exportaciones y una tendencia hacia una mayor diversificación de ellas. No obstante, como se ha visto en las secciones precedentes, el sector externo está aún fuertemente concentrado en un número reducido de mercados, productos y empresas. Esto pone de manifiesto la existencia de una serie de desafíos que deberán superarse para generar una estructura de exportaciones mucho más diversificada y, por lo mismo, menos expuesta a las fluctuaciones internacionales.

En este trabajo hemos analizado uno de los aspectos que pueden entregar antecedentes importantes para mejorar la inserción internacional de la economía chilena. Hemos hecho hincapié fundamentalmente en el comportamiento exportador de las empresas, con miras a identificar algunos de sus hechos estilizados y teniendo como acicate la existencia de un grupo considerable de firmas que no logran exportar permanentemente. En concordancia con la evidencia presenta$\mathrm{da}$, este problema de baja persistencia en la exportación está presente en la mayoría de los sectores exportadores, y es independiente del período bajo estudio y las fuentes de información utilizadas.

¿Por qué esto podría ser importante? Como se ha expuesto en este artículo, las empresas que exportan permanentemente lo hacen a más mercados y venden más productos en los mercados internacionales. A medida que nuevas firmas logren consolidarse como exportadoras, mayores serán las posibilidades de acrecentar la diversificación de las exportaciones chilenas.

Un segundo aspecto que surge de este estudio es el de las considerables diferencias que existen entre tipos de empresas. En general, y esto es consistente con una serie de estudios en otras economías, las empresas exportadoras exhiben mejores indicadores que las no exportadoras, en términos de tamaño, productividad, calificación de la mano de obra o innovación tecnológica. En este trabajo no sólo encontramos que tales diferencias existen en el caso chileno, sino también que hay diferencias importantes entre tipos de empresas exportadoras.

Aunque la evidencia presentada está lejos de representar una relación causal, estos hallazgos son consistentes con la idea de que el desempeño exportador está fuertemente relacionado con ciertas características de las empresas; en otras palabras, son sólo las "buenas" empresas las que logran exportar y permanecer en los mercados internacionales. Así, al formular políticas tendientes a mejorar la inserción internacional de la economía chilena es preciso tener presente que ciertas características de las empresas, como la productividad, el capital humano y la innovación tecnológica, son factores decisivos para el futuro éxito en la actividad exportadora, y que el mejoramiento de estas variables es condición necesaria para elevar el desempeño exportador.

\section{Bibliografía}

Agosin, M. (1999): Comercio y crecimiento en Chile, Revista de la CEPAL, $\mathrm{N}^{\circ}$ 68, LC/G.2039-P, Santiago de Chile, agosto.

Álvarez, R. y G. Crespi (2000a): Efecto de las políticas de fomento en el dinamismo exportador chileno, El trimestre económico, vol. 67(4), Nº 268, México, D.F., Fondo de Cultura Económica, octubre-diciembre.

(2000b): Exporter performance and promotion instruments: Chilean empirical evidence, Estudios de economía, vol. 27, $\mathrm{N}^{\circ}$ 2, Santiago de Chile, Universidad de Chile, diciembre.

Álvarez, R. y A. Lemus (2001): ¿Se ha detenido el proceso de diversificación de las exportaciones chilenas durante los noventa?, Estadística y economía, $\mathrm{N}^{\circ} 21$, Santiago de Chile, Instituto Nacional de Estadísticas (INE)

Aw, B.Y, S. Chung y M. Roberts (1998): Productivity and the Decision to Export: Micro Evidence from Taiwan and South
Korea, Working Paper, № 6558, Cambridge, Massachusetts, National Bureau of Economic Research (NBER), mayo.

Baldwin, J.R. y W. Gu (2003): Export-market participation and productivity performance in Canadian manufacturing, The Canadian Journal of Economics, vol. 36, $\mathrm{N}^{\circ} 3$, Oxford, Blackwell Publishing.

Banco Mundial (1996): Micro Foundations of Successful Export Promotion, RPO 679-20, Washington, D.C.

Bernard, A. y J.B. Jensen (2001): Why Some Firms Export?, Working Paper, $N^{\circ}$ 8349, Cambridge, Massachusetts, National Bureau of Economic Research (NBER), julio.

(1999): Exceptional performance exporter: cause, effect or both?, Journal of International Economics, vol. 47, $\mathrm{N}^{\circ} 1$ Madison, Wisconsin, University of Wisconsin. 
Bernard, A. y J. Wagner (2001): Export entry and exit by German firms, Weltwirtschaftliches Archiv, vol. 37, № 1, Kiel, Kiel Institute for World Economics.

Clerides, S., S. Lach y J. Tybout (1998): Is learning by exporting important? Micro-dynamic evidence from Colombia, Mexico, and Morocco, The Quarterly Journal of Economics, vol. 113, $\mathrm{N}^{\mathrm{o}}$ 3, Cambridge, Massachusetts, The MIT Press, agosto.

DIRECON (Dirección General de Relaciones Económicas Internacionales) (1999): Informe de comercio exterior de Chile, Santiago de Chile, Ministerio de Relaciones Exteriores.

Ffrench-Davis, R. (2002): El impacto de las exportaciones sobre el crecimiento en Chile, Revista de la CEPAL, N ${ }^{\circ} 76$, LC/G.2175-P, Santiago de Chile, abril.

Ffrench-Davis, R. y R. Sáez (1995): Comercio y desarrollo industrial, Colección Estudios CIEPLAN, No 41, Santiago de Chile, Corporación de Investigaciones Económicas para Latinoamérica (CIEPLAN), diciembre.

Figueroa, L. y L. Letelier (1994): Exportaciones, orientación al comercio y crecimiento: un enfoque de cointegración, Cuadernos de economía, $\mathrm{N}^{\circ}$ 94, Santiago de Chile, Instituto de Economía, Pontificia Universidad Católica de Chile, diciembre.

Fuentes, R. (1995): Openness and economic efficiency: evidence from the Chilean manufacturing industry, Estudios de economía, vol. 22, número especial, Santiago de Chile, Universidad de Chile, diciembre.

García, P., P. Meller y A. Repetto (1996): Las exportaciones como motor del crecimiento: la evidencia chilena, en P. Meller (comp.), El modelo exportador chileno: crecimiento y equidad, Santiago de Chile, Corporación de Investigaciones Económicas para Latinoamérica (CIEPLAN), mayo.

Gutiérrez de Pinheres, S. y M. Ferrantino (1997): Export diversification and structural dynamics in the growth process: the case of Chile, Journal of Development Economics, vol. 52, № 2 , Amsterdam, Elsevier Science, abril.

Isgut, A. (2001): What's different about exporters? Evidence from Colombian manufacturing, The Journal of Development Studies, vol. 37, No 5, Londres, Frank Cass Publishers, junio.

Macario, C. (2000): Chile: In search of a new export drive, en C. Macario (comp.), Export Growth in Latin America: Policies and Performance, Boulder, Colorado, Lynne Rienner Publishers/Comisión Económica para América Latina y el Caribe (CEPAL).

Marshall, I. (1992): Liberalización comercial en Chile y su impacto sobre la eficiencia técnica industrial: 1974-1986, Colección de Estudios CIEPLAN, $\mathrm{N}^{\circ}$ 35, Santiago de Chile, Corporación de Investigaciones Económicas para Latinoamérica (CIEPLAN), septiembre.

ProChile (Dirección de Promoción de Exportaciones) (2000): Análisis de las exportaciones chilenas, Santiago de Chile, Ministerio de Relaciones Exteriores.

(2002): Análisis de las exportaciones chilenas, Santiago de Chile, Ministerio de Relaciones Exteriores.

Roberts, M. y J. Tybout (1997): The decision to export in Colombia: an empirical model of entry with sunk costs, The American Economic Review, vol. 87, No 4, Nashville, Tennessee, American Economic Association.

Rojas, P., E. López y S. Jiménez (1997): Determinantes del crecimiento y estimación del producto potencial en Chile: el rol del comercio internacional, en F. Morandé y R. Vergara (comps.), Análisis empírico del crecimiento en Chile, Santiago de Chile, Centro de Estudios Públicos (CEP)

Tybout, J., J. De Melo y V. Corbo (1991): The effects of trade reforms on scale and technical efficiency: new evidence from Chile, Journal of International Economics, vol. 31, Madison, Wisconsin, University of Wisconsin. 